\title{
CONTROL OF A 2-DOF INVERTED PENDULUM ON AN OMNI- DIRECTIONAL PLATFORM
}

\author{
Tao SONG', Qibo JIA2, Shuai GUO3, Wei DAI ${ }^{4}$, Fengfeng (Jeff) XI ${ }^{5}$ \\ 1,2,3,4 Key Laboratory of Intelligent Manufacturing and Robotics, School of Mechatronics, Engineering and \\ Automation, Shanghai University, HC204, No. 99, Road Shangda, Shanghai 200444, China \\ ${ }^{5}$ Department of Aerospace Engineering, Ryerson University, 350 Victoria Street, Toronto, ON M5B 2K3, Canada \\ E-mail: songtao43467226@shu.edu.cn,1731977106@qq.com, guoshuai@shu.edu.cn, david1021@qq.com \\ fengxi@ryerson.ca
}

\begin{abstract}
This paper presents a method for the control of a 2-DOF inverted pendulum mounted on an omni-directional mobile platform. The system dynamics model is first established, and then two controllers are designed considering the acceleration and motor torque as two different inputs. Simulation is then carried out to compare their performances. It can be found that the torque controller is better than the acceleration controller. Two sets of experiment are conducted to show that the system can reach a stable state within $200 \mathrm{~ms}$.
\end{abstract}

Keywords: Omni-directional Platform, Inverted Pendulum, Mecanum wheel, LQR Control.

\section{Introduction}

The mobile platforms with Mecanum wheels (MPMW) can move in any direction with sharp turns. For this advantage, there is a rising interest in research of this omni-directional mobile platforms. MohdSalih and Rizon were the first ones who constructed a DC motor-driven MPMW and validated its performance of omni-direction movement [1]. Later, Chen and Fan proposed a kinematic model for the design and control of MPMW [2]. Hou and Li demonstrated an application of MPMW for forklift [3]. Lu and Cong designed a mobile platform using stepper motors and analysed the torque performance [4]. Wang proposed the dynamics equations for the control of MPMW [5]. Shi and Kang developed a MPMW for patient transfer [6].

As far as the motion of the omni-directional mobile platform is concerned, there are three degrees of freedom (DOFs) in plane, i.e. the translation in the $\mathrm{X}$ and $\mathrm{Y}$ direction and the rotation in $\mathrm{Z}$ direction. The said motion is realized by the differential speeds of the four-wheels. This character offers great convenience for the motion control and provides a fast response time, compared with traditional wheeled mobile platform which uses steering and driving control. However, MPMW has some shortcomings, for instant, higher requirement on surface flatness and torque imbalance in wheel motors. In particular, when a MPMW climbs up a ramp of $45^{\circ}$, only the front two wheels apply torque while the rear two do not. Due to this load imbalance, large torque motors are usually needed for MPMW. This imbalance also implies that some special measure should be taken for the motor control in order to ensure that the motor torques for a specific task stay within the allowed maximum values.

This paper aims at addressing the motor torque issue. For this purpose, an inverted pendulum mounted on a MPMW is used to study how to minimize the motor torque while ensuring the pendulum stability. The system model is established including an inverted pendulum mounted on the MPMW. A LQR controller is designed to minimize the motor torques. In what follows, the details are provided.

\section{System Description}

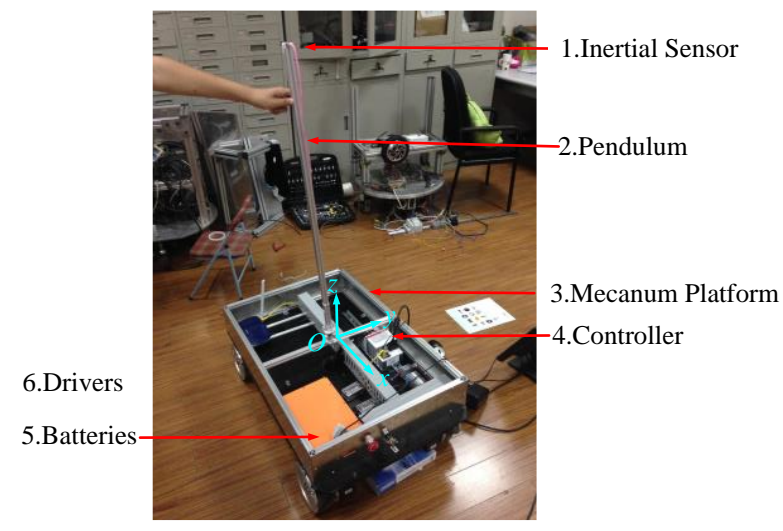

Figure 1: The MPMW with an inverted pendulum

Figure 1 shows the experiment system developed at Shanghai University where an inverted pendulum is mounted onto an omni-directional mobile platform. The inverted pendulum is connected to the platform through a universal joint. The inertial sensor is fixed 
on the top of the pendulum to measure the rotation angle and the angular velocity of the pendulum. Four Mecanum wheels are driven by four stepper motors. The position and velocity of the mobile platform are calculated by the counts and frequency of the impulses sent by the controller to the driver.

\section{Dynamics of the MPMW}

\subsection{The mathematical model of inverted pendulum system}

Figure 2 shows the coordinate system attached to the mobile platform, and it is denoted as $\{O X Y Z\}$. The two rotation axes for the pendulum are denoted by $\mathrm{u}_{\mathrm{x}}$ and $\mathrm{u}_{\mathrm{y}}$ about $\mathrm{X}$ and $\mathrm{Y}$ axis respectively. The angle between the pendulum and vertical $\mathrm{Z}$ axis is called the intersection angle and denoted by $\theta$. The angle between the projection of the pendulum in X-Y plane and $\mathrm{Z}$ axis is called the yaw angle and denoted by $\alpha$, with the counter clockwise direction as its positive direction.

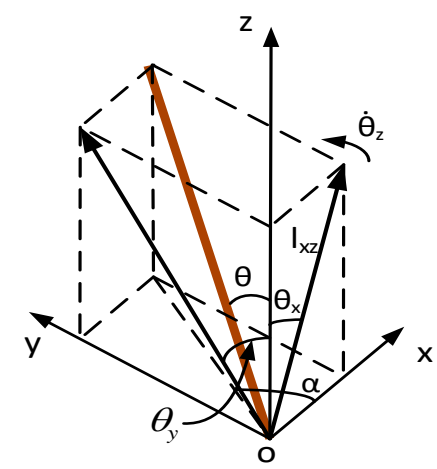

Figure 2: Intersection angle and yaw angle in the system

The rotation axis $\mathrm{u}_{\mathrm{s}}$ is along $\mathrm{X}$ axis and the rotation axis $\mathrm{u}_{y}$ is along $\mathrm{Y}$ axis which will change after the rotation about $X$ axis. These two angles are denoted by $\theta_{x}, \theta_{y}$, respectively. When the intersection angle is small, the inverted pendulum dynamics can be approximated as two single DOF inverted pendulums in $\mathrm{X}-\mathrm{Z}$ and $\mathrm{Y}-\mathrm{Z}$ plane, respectively [7]. Without loss of generality, the situation in $X-Z$ plane is always considered first. In this plane, the kinetic energy $\mathrm{T}$ and potential energy $V$ are shown below:

$$
\left\{\begin{array}{l}
\mathrm{T}=\int_{0}^{1} \rho\left(\frac{1}{2} \mathrm{~s}^{2} \cdot \sin ^{2} \theta_{\mathrm{x}} \cdot \theta_{\mathrm{z}}^{2}+\frac{1}{2} \mathrm{~s}^{2} \theta_{\mathrm{x}}^{2}+\right. \\
\left.\frac{1}{2} \mathrm{v}_{\mathrm{x}}^{2}+\mathrm{s} \cdot \cos \theta_{\mathrm{x}} \cdot \theta_{\mathrm{x}} \cdot \mathrm{v}_{\mathrm{x}}\right) \mathrm{ds} \\
\mathrm{V}=\frac{1}{2} \mathrm{mgl \operatorname {cos } \theta _ { \mathrm { x } }}
\end{array}\right.
$$

There are two equations for the generalized forces in two generalized coordinates by assuming that the friction coefficient is zero.

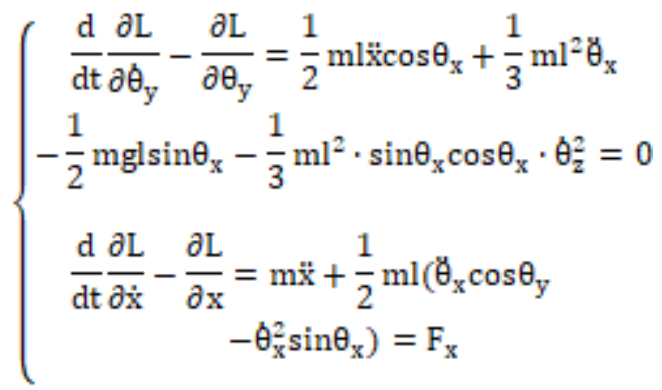

The system model is linearized with an equilibrium position at $x=0, \theta=0$. The nonlinear term is negligible because the rotational speed about $\mathrm{Z}$ axis is small. The influence of the inverted pendulum on the whole system is three order infinitesimal when the intersection angle and the rotational speed are both small. In this case,

$$
\left\{\begin{array}{c}
\mathrm{F} \approx \mathrm{mx}+\frac{1}{2} \mathrm{ml}_{\mathrm{x}} \\
\mathrm{T}_{\text {Torque }} \approx \frac{1}{3} \mathrm{ml}^{2} \mathrm{\theta}-\frac{1}{2} \mathrm{ml} \overline{\mathrm{x}}-\frac{1}{2} \mathrm{mgl}
\end{array}\right.
$$

The state-space expressions near $\mathrm{x}=0, \theta_{\mathrm{x}}=0$ are shown in Eqn. (4)

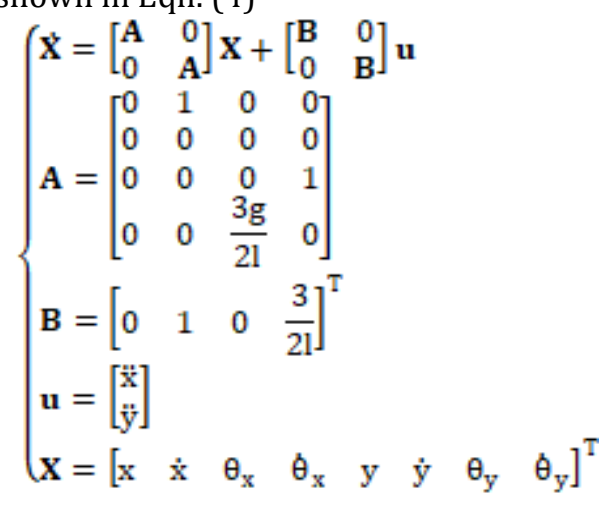

\subsection{Kinematics and dynamics model of the omni-direction platform}

The dynamics equations of the MPMW can be obtained as follow [8-10].

$$
\left[\begin{array}{l}
\bar{x} \\
\bar{y} \\
\bar{\theta}
\end{array}\right]=R_{\omega} J^{+} M^{-1} \tau
$$

$$
\begin{aligned}
& M=\left[\begin{array}{cccc}
A_{T}+B_{T}+I_{\omega} & -B_{T} & B_{T} & A_{T}-B_{T} \\
-B_{T} & A_{T}+B_{T}+I_{\omega} & A_{T}-B_{T} & B_{T} \\
B_{T} & A_{T}-B_{T} & A_{T}+B_{T}+I_{\omega} & -B_{T} \\
A_{T}-B_{T} & B_{T} & -B_{T} & A_{T}+B_{T}+J_{\Omega}
\end{array}\right] \\
& A_{T}=\frac{m^{s} R_{\omega}^{2}}{8}{ }_{v} B_{T}=\frac{J_{z} R_{\omega}^{2}}{16\left(L_{1}+L_{2}\right)}
\end{aligned}
$$

The Jacobian matrix of the MPMW can be obtained as 


$$
\mathbf{J}=\left[\begin{array}{ccc}
1 & -1 & -\left(\mathrm{L}_{1}+\mathrm{L}_{2}\right) \\
1 & 1 & \left(\mathrm{~L}_{1}+\mathrm{L}_{2}\right) \\
1 & 1 & -\left(\mathrm{L}_{1}+\mathrm{L}_{2}\right) \\
1 & -1 & \left(\mathrm{~L}_{1}+\mathrm{L}_{2}\right)
\end{array}\right]
$$

The generalized inverse of the Jacobian matrix can be obtained as well.

$$
I^{\oplus}=\frac{1}{4}\left[\begin{array}{cccc}
1 & 1 & 1 & 1 \\
-1 & 1 & 1 & -1 \\
-\frac{1}{\left(L_{1}+L_{2}\right)} & \frac{1}{\left(L_{1}+L_{2}\right)} & -\frac{1}{\left(L_{1}+L_{2}\right)} & \frac{1}{\left(L_{1}+L_{2}\right)}
\end{array}\right]
$$

The parameters of the mobile platform under this study are listed in Table 1.

\begin{tabular}{|c|c|c|}
\hline Symbol & Name & Value \\
\hline $\mathbf{M}$ & \multicolumn{2}{|c|}{ Mass matrix } \\
\hline $\mathbf{J}$ & \multicolumn{2}{|c|}{ Jacobian matrix } \\
\hline $\mathrm{J}^{+}$ & $\begin{array}{c}\text { Generalized inverse matrix of the } \\
\text { Jacobian }\end{array}$ \\
\hline $\mathrm{m}^{\prime}$ & Mass of the system & $109.3 \mathrm{~kg}$ \\
\hline $\mathrm{l}$ & Pendulum length & $1000 \mathrm{~mm}$ \\
\hline $\mathrm{L} 1$ & Half axle & $350 \mathrm{~mm}$ \\
\hline $\mathrm{L} 2$ & Half track & $250 \mathrm{~mm}$ \\
\hline $\mathrm{R}_{\mathrm{w}}$ & Radius of wheel & $82.5 \mathrm{~mm}$ \\
\hline $\mathrm{J}_{\mathbf{z}}$ & $\begin{array}{c}\text { Moment of Inertial } \\
\text { around Z Axis }\end{array}$ & 6.2179 \\
\hline $\mathrm{J}_{\omega}$ & Wheel inertial moment & $0.029 \mathrm{~kg}{ }^{2} \mathrm{~m}^{2}$ \\
\hline
\end{tabular}

\subsection{The Dynamics model of the system}

A few assumptions are made to simplify the analysis. The influence of the gravity and inertial force of the inverted pendulum on the MPMW are negligible, and the friction between the wheels and ground is regarded as static one. Now, we can have the following equations:

$$
\left\{\begin{array}{l}
\tau_{1}=\tau_{4} \\
\tau_{2}=\tau_{3}
\end{array}, \quad\left[\begin{array}{l}
\tilde{x} \\
\bar{y} \\
\bar{\theta}
\end{array}\right]=R_{\omega} J^{+} \mathbf{M}^{-1} \tau\right.
$$

Let the parameters be satisfied:

$$
\begin{aligned}
& {\left[\begin{array}{l}
\bar{x}_{\mathrm{W}} \\
\underline{y}_{\mathrm{W}}
\end{array}\right]=\mathbf{P}\left[\begin{array}{l}
\mathrm{x} \\
\bar{y} \\
\bar{\theta}
\end{array}\right]} \\
& P=\left[\begin{array}{lll}
1 & 0 & 0 \\
0 & 1 & 0
\end{array}\right] \\
& \left\{\begin{array}{l}
\tau_{1}=\tau_{4}=\tau_{A} \\
\tau_{2}=\tau_{3}=\tau_{B}
\end{array}\right.
\end{aligned}
$$

So, the simultaneous equation can be obtained with Eqn. (4)
Table 1. Platform parameters

$$
\mathbf{X}=\left[\begin{array}{ll}
A & 0 \\
0 & A
\end{array}\right] \mathbf{X}+\left[\begin{array}{ll}
B & 0 \\
0 & B
\end{array}\right] P R_{W} J^{+} \mathbf{M}^{-1}\left[\begin{array}{c}
\tau_{A} \\
\tau_{B}
\end{array}\right]
$$

The equations for the omni-directional platform with an inverted pendulum are:

$$
\begin{aligned}
\mathbf{x} & =\left[\begin{array}{llllllll}
\mathrm{x} & \tilde{\mathrm{x}} & \theta_{\mathrm{x}} & \theta_{\mathrm{x}} & \mathrm{y} & \dot{\mathrm{y}} & \theta_{y} & \theta_{y}
\end{array}\right]^{\mathrm{T}} \\
\mathbf{u}^{\prime} & =\left[\begin{array}{l}
\tau_{\mathrm{A}} \\
\tau_{\mathrm{B}}
\end{array}\right]
\end{aligned}
$$

$$
\left\{\begin{array}{l}
\mathbf{A}^{v}=\left[\begin{array}{ll}
\mathbf{A} & 0 \\
0 & \mathrm{~A}
\end{array}\right] \\
\mathbf{B}^{v}=\left[\begin{array}{ll}
\mathbf{B} & 0 \\
0 & \mathbf{B}
\end{array}\right] \mathrm{PR}_{\omega} \mathbf{J}^{+} \mathbf{M}^{-1}
\end{array}\right.
$$

At last, the standard form can be obtained

$$
\mathbf{X}=\mathbf{A}^{t} \mathbf{X}+\mathbf{B}^{f} \mathbf{u}^{f}
$$

\section{LQR Controller Design}

\subsection{Controller design with torque minimization}

A LQR controller is a type of the state feedback controller based on the optimal control theory. The goal is to minimize the objective function which is constructed in the form as $\mathrm{I}(\mathbf{u})=\int_{0}^{\mathrm{s}}\left(\mathbf{x}^{\mathrm{T}} \mathbf{Q} \mathbf{x}+\mathbf{u}^{\mathrm{T}} \mathbf{R u}\right) \mathrm{dt}$ where the $\mathbf{Q}$ and $\mathbf{R}$ is the weight matrix of states and inputs, respectively. The system dynamics parameters are known such as $\mathbf{A}^{*}$, $\mathbf{B}^{x}$. To minimize the motor torques, the system should maximize $\mathbf{R}$ and minimize $\mathbf{Q}$ at the same time. Here, $\mathbf{R}$ and $\mathbf{Q}$ are set as below.

$$
\mathbf{Q}^{s}=\left[\begin{array}{llll}
1 & 0 & 0 & 0 \\
0 & 1 & 0 & 0 \\
0 & 0 & 1 & 0 \\
0 & 0 & 0 & 1
\end{array}\right]
$$

$$
\begin{aligned}
& \mathbf{R}=\left[\begin{array}{cccc}
10000 & 0 & 0 & 0 \\
0 & 10000 & 0 & 0 \\
0 & 0 & 10000 & 0 \\
0 & 0 & 0 & 10000
\end{array}\right] \\
& \mathbf{Q}=\left[\begin{array}{cc}
\mathbf{Q}^{s} & 0 \\
0 & \mathbf{Q}^{s}
\end{array}\right]
\end{aligned}
$$

The feedback matrix $\mathbf{K}^{t}$ is given as:

$$
\mathbf{K}=\left[\begin{array}{cccccccc}
- \text {-uwusz } & - \text {-uvez } & 20 u v 1 & b .220 & 0 & 0 & 0 & 0 \\
0 & 0 & 0 & 0 & -0.0032 & -0.082 & 20.01 & 5.220
\end{array}\right]
$$




\subsection{LQR controller design for acceleration minimization}

When the motor torques are applied, the platform will produce an acceleration as shown in Eqn. (5). It provides another way to reduce the motor torques by reducing the mobile platform's acceleration. The second controller is designed here with this purpose. In this case, the inputs are two accelerations along the $\mathrm{X}$ and $\mathrm{Y}$ axis as shown in Eqn. (4). To compare the performances between the two controllers, the values of the weight matrix $\mathbf{Q}$ and $\mathbf{R}$ are kept the same. The state feedback matrix $\mathbf{K}_{\text {acc }}$ based on the acceleration optimization is calculated as

$$
K_{\operatorname{man}}=\left[\begin{array}{cccccccc}
-0.01 & -0.1 .15 & 20.3 & b .3 & 0 & 0 & 0 & 0 \\
0 & 0 & 0 & 0 & -0.01 & -0.15 & 20.3 & 5.3
\end{array}\right]
$$

\section{Simulation Analysis 5.1 Simulation Model}

A Simulink ${ }^{\circledR}$ model of the MPMW with inverted pendulum system is established as shown in Figure 3.

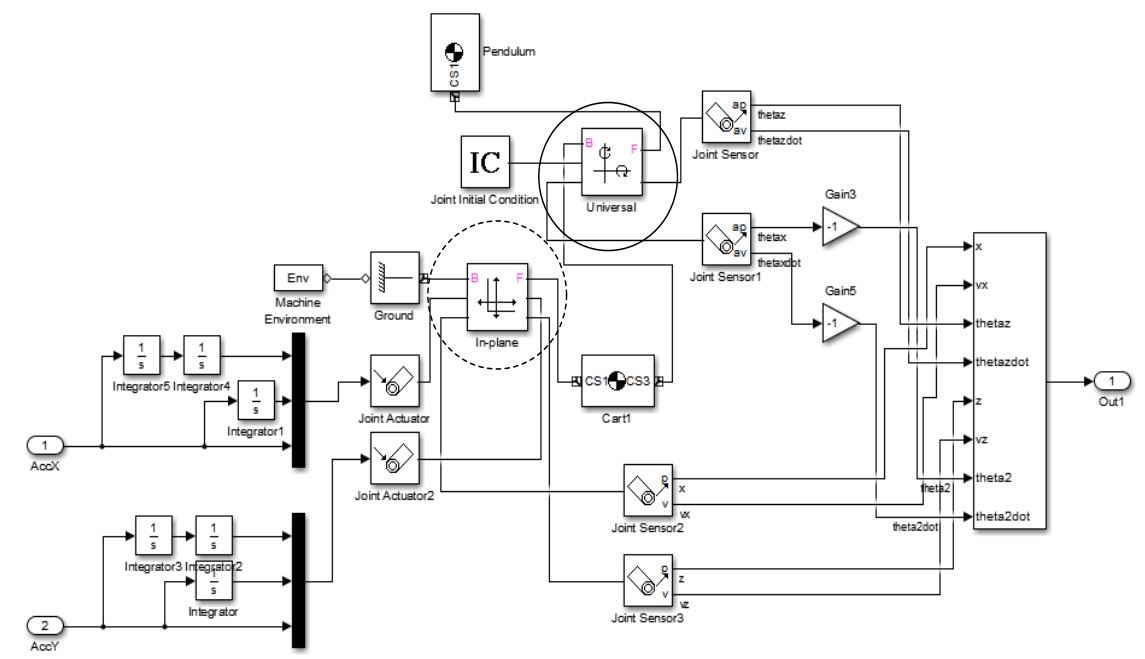

Figure 3: Dynamics model of the pendulum

The pendulum is connected through the universal joint to the MPMW (as shown with solid line). There is no rotation of the platform on the ground, and the motion of the platform is constrained in translational motion on the ground (as shown with dashed line) only.

\subsection{The result of motor torques optimization}

The system designed above was simulated for the yaw angle from $0^{\circ}$ to $360^{\circ}$ and the intersection angles are set as $2^{\circ}, 4^{\circ}, 6^{\circ}, 8^{\circ}$ and $10^{\circ}$. Figure 4 shows the torque curve in terms of the pendulum's yaw angle.

Each curve in Figure 4 represents the maximum torque in different yaw angles. The curves from inward to outward are drawn with the intersection angle from $2^{\circ}$ to $10^{\circ}$. The torque value increases with the increasing intersection angle under the same yaw angle. The maximum torque value increases with the yaw angle from $0^{\circ}$ to $45^{\circ}$, but decreases with the yaw angle from $45^{\circ}$ to $90^{\circ}$.

The accelerations to move the pendulum back to the equilibrium position are roughly the same when the pendulum has a same intersection angle.

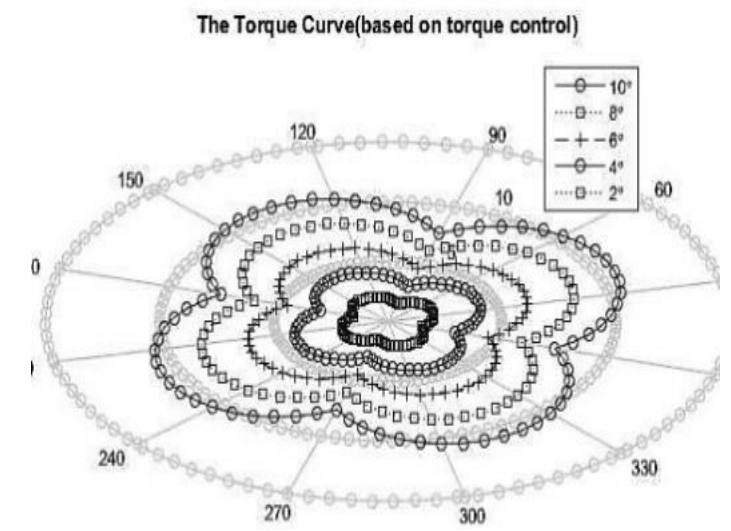

Figure 4: Maximum torque curve

If a denotes the linear acceleration, the acceleration components can be obtained as:

$\left\{\begin{array}{l}\mathrm{X}=a \cos \alpha \\ \frac{\mathrm{m}}{\mathrm{y}}=\mathrm{a} \sin \alpha\end{array}\right.$

Combining it with Eqn. (5), it can yield

$$
\left[\begin{array}{c}
\operatorname{acos} \alpha \\
\operatorname{asin} \alpha \\
0
\end{array}\right]=R_{\omega} J^{+} \mathbf{M}^{-1} \mathbf{\tau}
$$


After simplification, it can be transferred to

$$
\left[\begin{array}{l}
\tau_{A} \\
\tau_{B} \\
\tau_{B} \\
\tau_{A}
\end{array}\right]=\frac{\left(2 A_{D}+J_{\omega}\right)}{R_{\omega}}\left[\begin{array}{l}
\sqrt{2} \operatorname{acos}\left(\alpha+\frac{\pi}{4}\right) \\
\sqrt{2} \operatorname{asin}\left(\alpha+\frac{\pi}{4}\right) \\
\sqrt{2} \operatorname{asin}\left(\alpha+\frac{\pi}{4}\right) \\
\sqrt{2} \operatorname{acos}\left(\alpha+\frac{\pi}{4}\right)
\end{array}\right]
$$

where $\tau_{A}$ and $\tau_{B}$ are trigonometric functions and the period is $2 \pi$. The phase difference is $\pi / 4$ indicating the maximum torque.

The maximum values of the four motor torque corresponding to a certain intersection angle are listed in the Table 2.

Table 2. Torque values under different intersection angle

\begin{tabular}{|c|c|c|c|c|c|}
\hline $\begin{array}{c}\text { Intersection Angle } \\
\left({ }^{\circ}\right)\end{array}$ & 2 & 4 & 6 & 8 & 10 \\
\hline$\tau_{\max }(\mathrm{N} \times \mathrm{m})$ & 2.1 & 4.3 & 6.5 & 8.7 & 10.8 \\
\hline$\tau_{\min }(\mathrm{N} \times \mathrm{m})$ & 1.5 & 3.1 & 4.6 & 6.1 & 7.7 \\
\hline
\end{tabular}

The maximum torque is beyond the motor static torque when the intersection angle is larger than $6^{\circ}$. The stable range of the intersection angle is between $0^{\circ}$ to $6^{\circ}$.

\subsection{The result of acceleration optimization}

With the same intersection and yaw angle, the simulation result of the acceleration input optimization is presented in Figure 5 . The polar angle is the pendulum's yaw angle and the polar radius is the maximum torque of the four wheels.

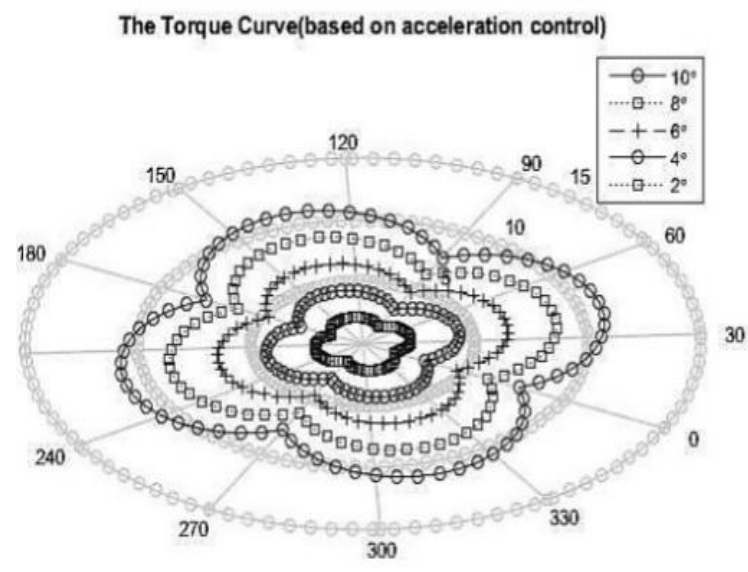

Figure5: Maximum torque about yaw angle curve

Comparing it with Figure 4, the two torque are same, as analysed above. The maximum value of the four motor torques in a certain intersection angle are given in Table 3.

Table 3. Torque values under different intersection

\begin{tabular}{|c|c|c|c|c|c|}
\hline $\begin{array}{c}\text { Intersection Angle } \\
\left({ }^{\circ}\right)\end{array}$ & 2 & 4 & 6 & 8 & 10 \\
\hline$\tau_{\max }(\mathrm{N} \cdot \mathrm{m})$ & 2.2 & 4.4 & 6.6 & 8.8 & 11.0 \\
\hline$\tau_{\min }(\mathrm{N} \times \mathrm{m})$ & 1.6 & 3.1 & 4.7 & 6.2 & 7.8 \\
\hline
\end{tabular}

The motor torque value of the optimization based on torque is larger than the optimization based on acceleration, because the weight is different in the two methods. Eqn. 25 can be simplified as

$$
\left[\begin{array}{l}
\tau_{A} \\
\tau_{B}
\end{array}\right]=\frac{\left(2 A_{I}+J_{\omega}\right)}{R_{\omega}}\left[\begin{array}{cc}
1 & -1 \\
1 & 1
\end{array}\right]\left[\begin{array}{l}
\mathrm{I} \\
\bar{y} \\
\bar{y}
\end{array}\right]
$$

The forms of acceleration input and torque input are linearly dependent. The objective function for both is the same $\mathbf{x}^{\mathbf{T}} \mathbf{Q x}$, but the input term $\mathbf{u}^{\mathbf{T}} \mathbf{R u}$ is different. The input weight matrix is $\mathbf{R}$, so $\mathbf{R}$ can be written as follows by the symmetry:

$$
\mathbf{R}=\left[\begin{array}{cc}
\mathrm{f} & \mathrm{k} \\
\mathrm{k} & \mathrm{f}
\end{array}\right](\mathrm{f}>\| \mathrm{k} \mid)
$$

Substituting (27) for $\mathbf{R}$ in (28) gives,

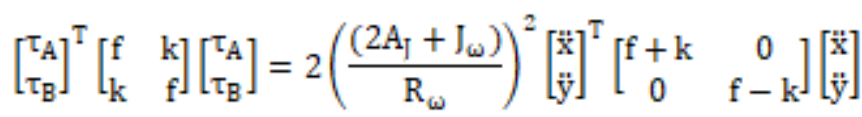

For the two optimization methods, the mathematics expression are linear dependent, meaning that the different control model can convert their input weight coefficients to have the same performance. Especially, when $\mathrm{k}=0$ : 


$$
\left[\begin{array}{l}
\tau_{A} \\
\tau_{B}
\end{array}\right]^{T}\left[\begin{array}{ll}
\mathrm{f} & 0 \\
0 & f
\end{array}\right]\left[\begin{array}{l}
\tau_{A} \\
\tau_{B}
\end{array}\right]=9.6214\left[\begin{array}{l}
\bar{x} \\
\bar{y}
\end{array}\right]^{T}\left[\begin{array}{ll}
\mathrm{f} & 0 \\
0 & f
\end{array}\right]\left[\begin{array}{l}
\mathrm{x} \\
\bar{y}
\end{array}\right]
$$

The two optimization methods only have different weight values. By changing the weight matrix value, the optimization based on acceleration will have the same performance as the optimization based on torque.

Bring the new weight matrix into the state-space Eqn. (4).

$$
\mathbf{R}=\left[\begin{array}{cc}
96214 & 0 \\
0 & 96214
\end{array}\right]
$$

The new state feedback matrix after optimization is:

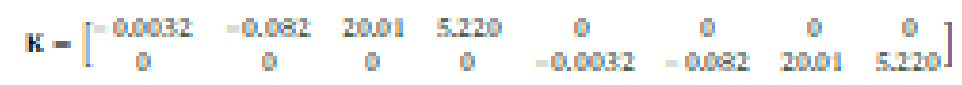

The values are the same as (22).

\section{Experiment}

A set of experiments showed the performance of the real-system, and the freedom of the pendulum in $\mathrm{Y}-\mathrm{Z}$ plane has been constrained to reduce the difficulty of

the experiment. In these experiments, the acceleration, orientations, and angular velocities of pendulum are measured and then the acceleration of the MPMW is calculated by the measured data.

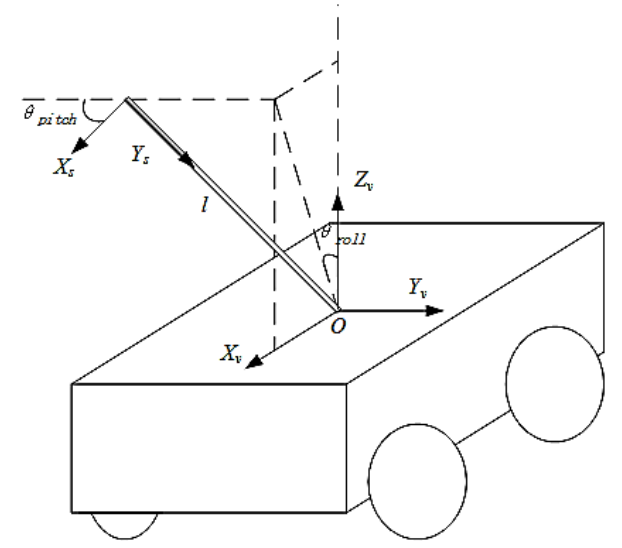

Figure 6: Sensor coordinate and the vehicle coordinate

The relationship of the measured data and the acceleration of the MPMW is shown in (23).

$$
\tilde{\mathbf{a}}_{\text {vehicle }}=\tilde{\mathbf{a}}_{\text {pendulum }}-\alpha \times \mathbf{r}-\omega^{2} \mathbf{r}
$$

The vector $\tilde{\mathbf{a}}_{\text {pendulum }}$ is the measured acceleration of

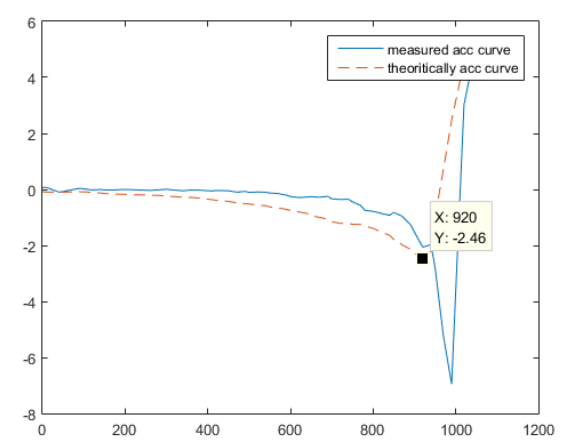

Figure 7: Accelerations curve of experiment 1 the pendulum, the vector $\tilde{\mathbf{a}}_{\text {vehicle is the calculated }}$ acceleration of the MPMW.

Each experiment has the recorded sensor data. To avoid the platform moving too fast to hit people, the velocity of the platform is limited below $2000 \mathrm{~mm} / \mathrm{s}$.

An emergency stop will be triggered automatically when the velocity over this limitation. The experiment data is shown in Figure 7 to 16.

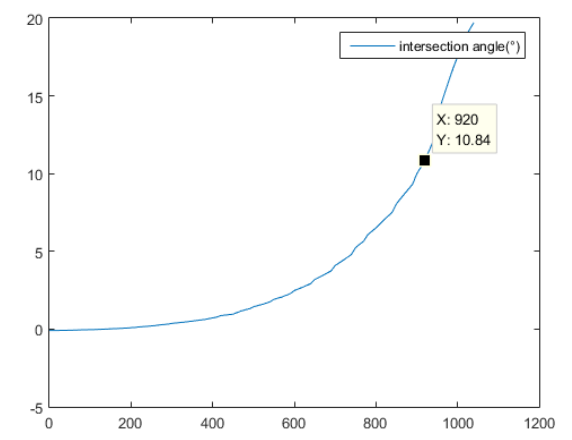

Figure 8: Intersection curve of experiment 1 


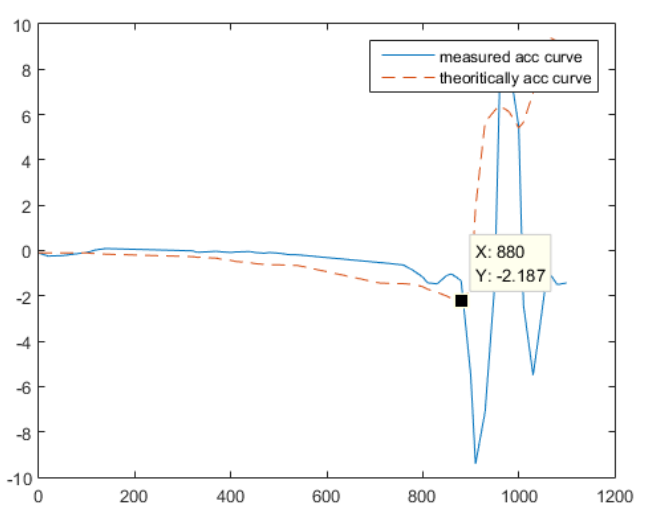

Figure 9: Accelerations curve of experiment 2

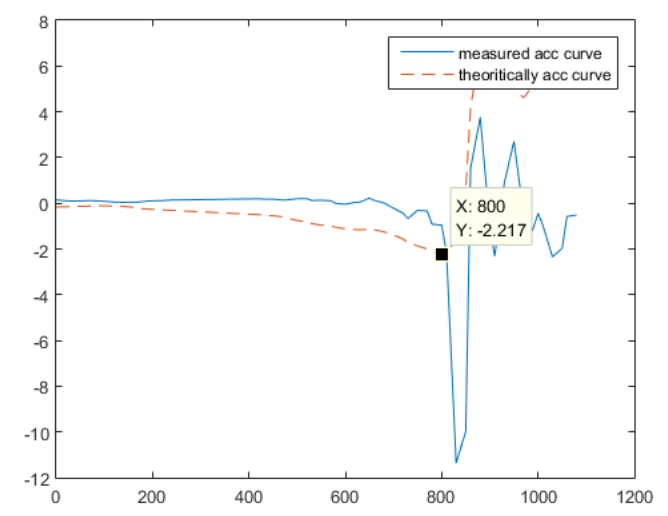

Figure 11: Accelerations curve of experiment 3

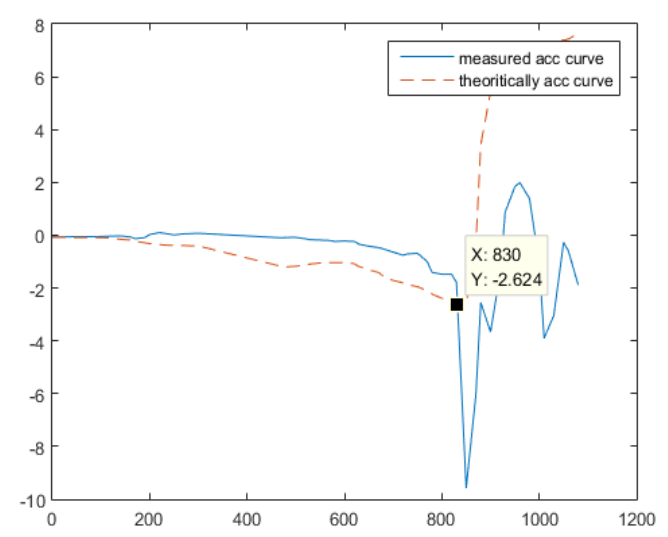

Figure 13: Accelerations curve of experiment 4

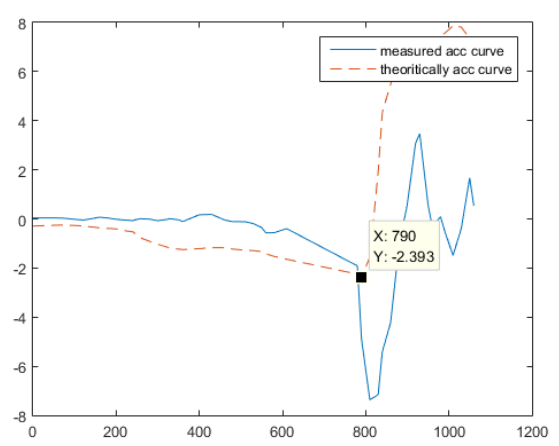

Figure 15: Accelerations curve of experiment 5

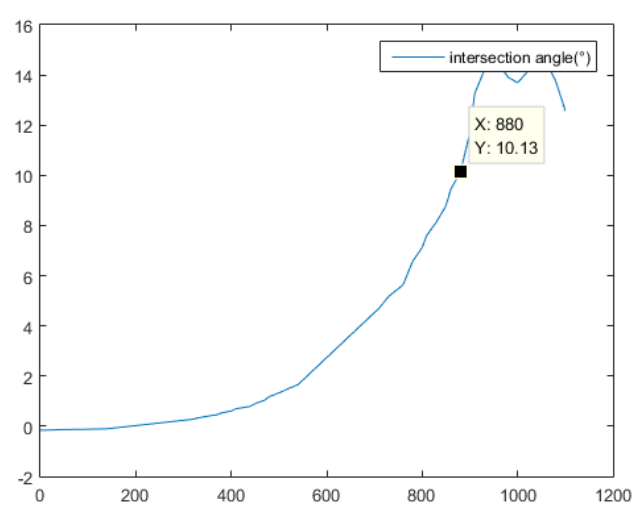

Figure 10: Intersection curve of experiment 2

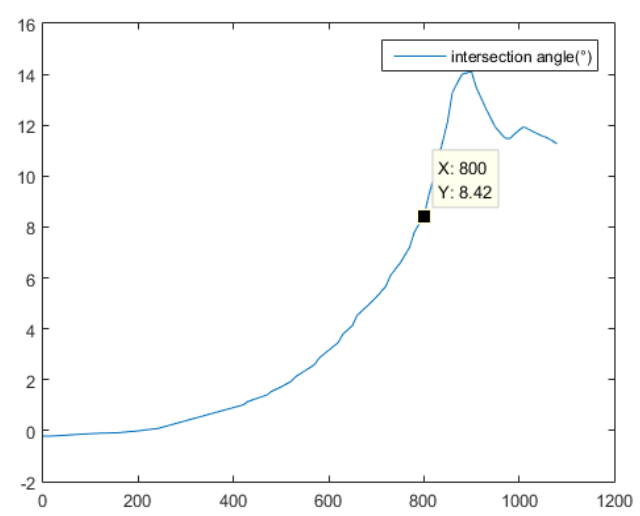

Figure 12: Intersection curve of experiment 3

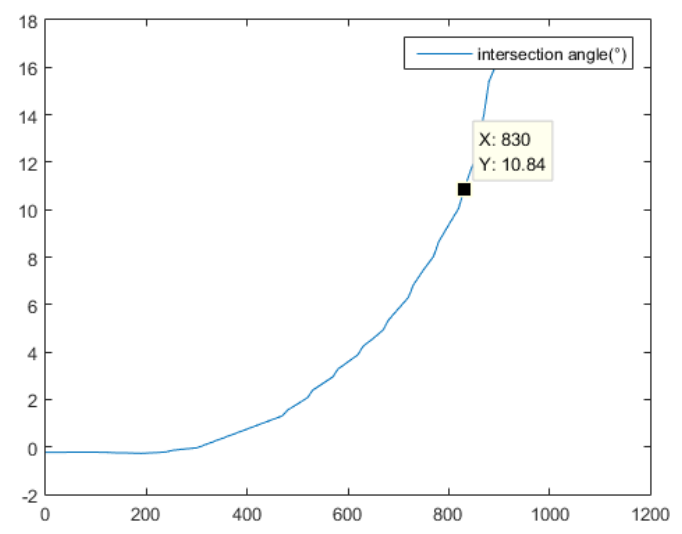

Figure 14: Intersection curve of experiment 4

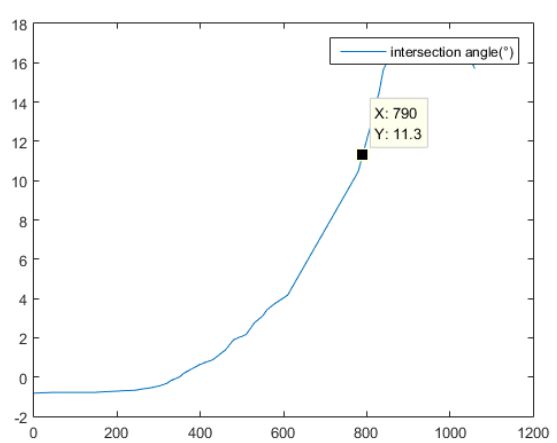

Figure 16: Intersection curve of experiment 5 
The time-intersection angle curve records the relationship between the intersection angle and the time. It can be found that the intersection angle increases with time and the system is unstable. In the time-acceleration curve, the red dotted line is the platform acceleration which was calculated based on the sensor data.. It can be seen that the change in the actual acceleration curve of the system is always lagging behind the theoretically acceleration curves.

The system acceleration curves become suddenly abrupt at $800 \mathrm{~ms}$, and the intersection angle at around 8 degrees. Theoretically it can be seen that the motor has been unable to produce enough torque to response the theoretical acceleration at this moment.

It can be found that the blue curves did not follow the red curves very well. There is a delay between the red curves and the blue curves.

By measuring the time interval between two sharp points (the first jump of these curves), the results are listed in Table 4.

Table 4.

Maximum torque values under different angle range

\begin{tabular}{|c|c|c|c|c|c|}
\hline Intersection Angle $\left(^{\circ}\right)$ & 2 & 4 & 6 & 8 & 10 \\
\hline Delay (ms) & 20 & 20 & 20 & 30 & 70 \\
\hline
\end{tabular}

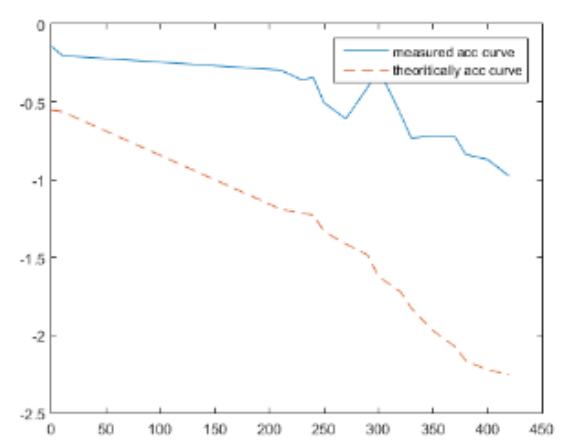

Figure 17: Accelerations curve of experiment 6

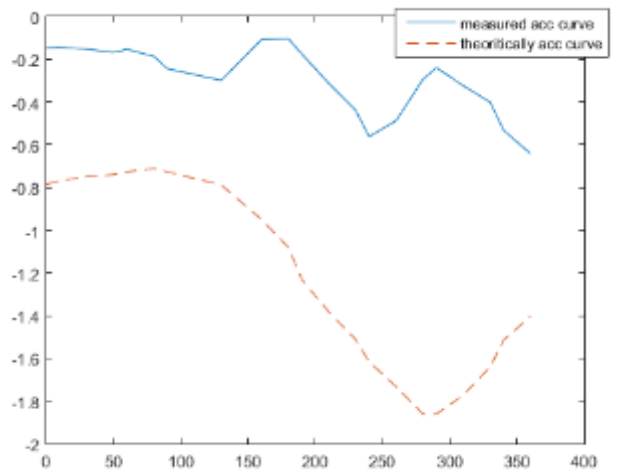

Figure 19: Accelerations curve of experiment 7
The average delay of the system is $32 \mathrm{~ms}$. The existence of system delay resulted in the instability of the system so the controller could not stabilize the pendulum in the vicinity of the equilibrium position.

There are several reasons which cause this system delay. First, the operation period in the controller computer is $10 \mathrm{~ms}$. Each time, the sensor signal changes, the current input signals processing requires $10 \mathrm{~ms}$ to read the sensor signals and calculate the output signals to drivers. Second, there is an electrical time constant, and the motor response is 5 to $10 \mathrm{~ms}$. At last, the friction effect results in the delay of the platform's response as well.

The first and second reasons are the inherent delay which cannot be changed, but the output torques could be increased by increasing the output values. Bigger motor torque may overcome influence of frictions, so the feedback coefficient is adjusted to twice the original value to make another set of experiment to observe the performance of the system with the results given in Figure 17 to 26.

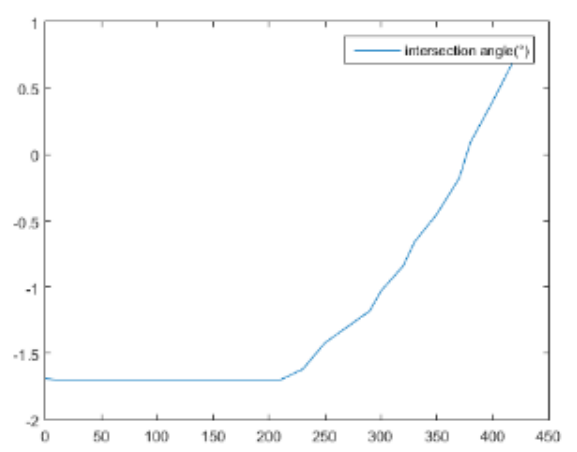

Figure 18: Intersection curve of experiment 6

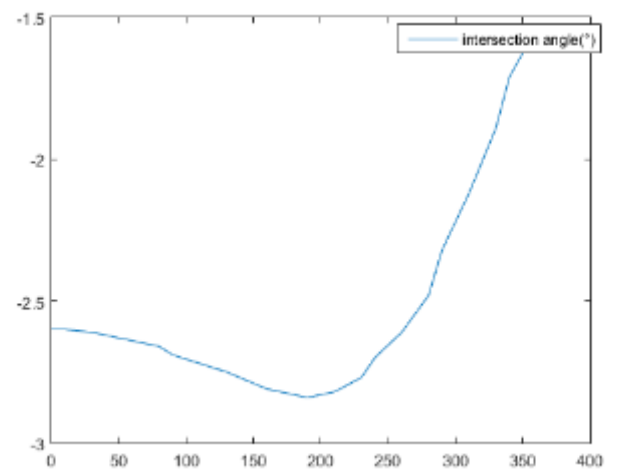

Figure 20: Intersection curve of experiment 7 


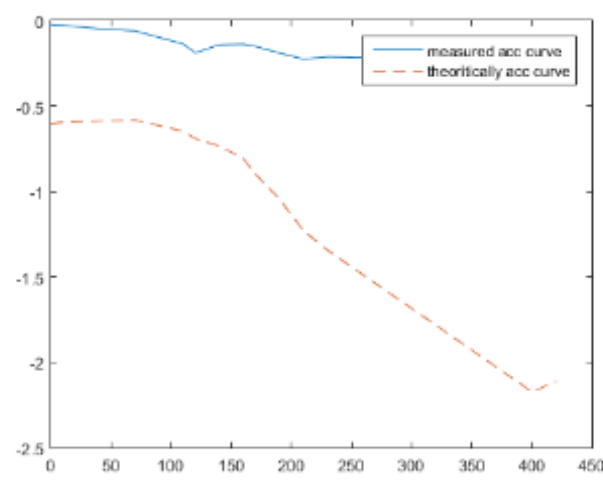

Figure 21: Accelerations curve of experiment 8

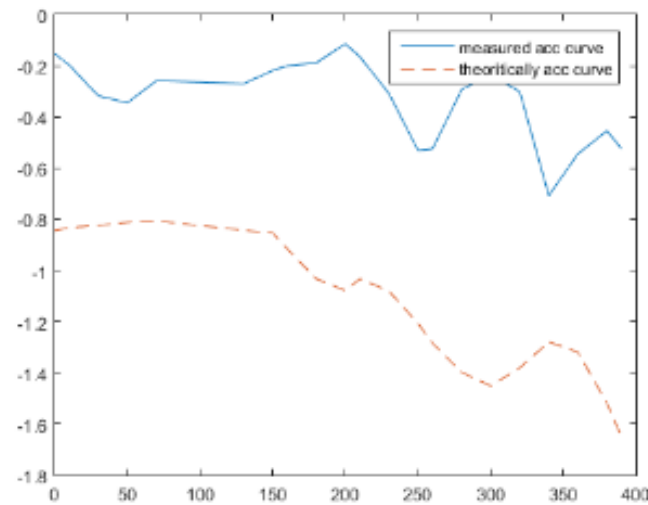

Figure 23: Accelerations curve of experiment 9

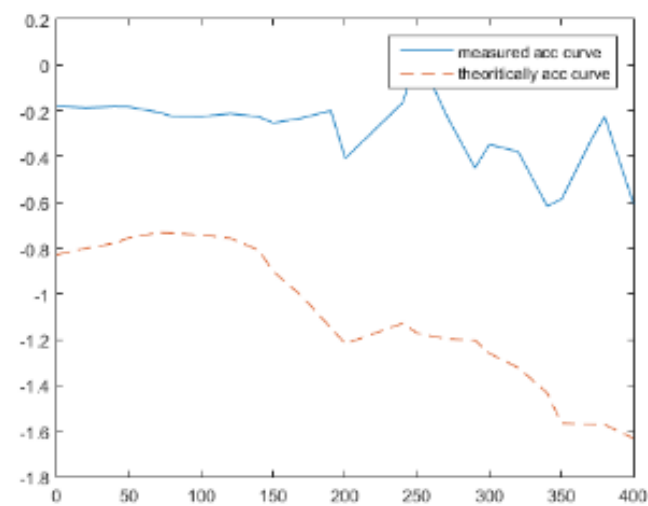

Figure 25: Accelerations curve of experiment 10

In this set of experiments, the delay of acceleration is reduced and the speed is faster than it in previous experiments. The change of acceleration is more violent. The reason is that the feedback coefficient is bigger than the previous one, and the stable time is $200 \mathrm{~ms}$, which is the same as the previous experiments.

\section{Conclusion}

Two LQR controllers are designed based on the optimization of the acceleration and torque for an inverted pendulum mounted on an omni-directional

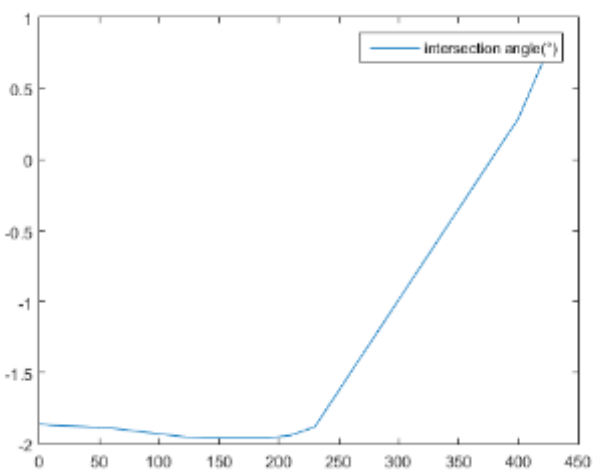

Figure 22: Intersection curve of experiment 8

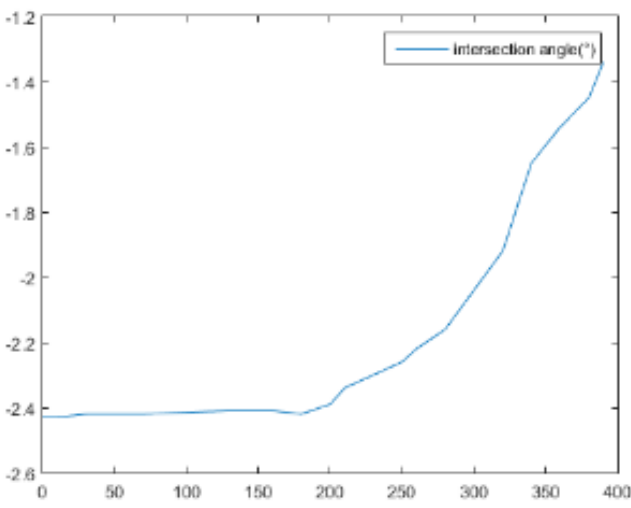

Figure 24: Intersection curve of experiment 9

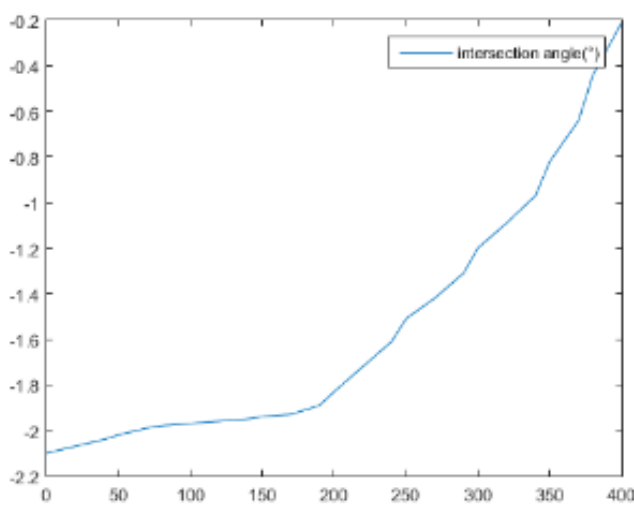

Figure 26: Intersection curve of experiment 10

mobile platform. The maximum torques of the motors have a four-leaves clover shaped distribution in the polar coordinate system when the intersection angle is constant. The motor torques and the acceleration of the platform are linearly dependent.

Changing the input weight matrix can obtain the same performance in different input models of LQR control.

This paper is focused on the performance in which the friction and the initial angular speed is negligible, and their effects will be studied in future research. Two sets of experiments are carried out to show the performance of the controllers. 
According to the results of the experiments, a conclusion has been obtained that system can reach a stable state within $200 \mathrm{~ms}$.

\section{Acknowledgement}

This paper is funded by the International Exchange Project (under Grant No. 15550721900 and 16DZ1120803) of Shanghai Municipal Commission of Science and Technology.

\section{References}

[1] Salih J E M, Rizon M, Yaacob S, et al. Designing omni-directional mobile robot with Mecanum wheel. American Journal of Applied Sciences, 2006, 3(5): 1831-1835.

[2] Chen Boweng, FAN Chuankang, HE Ji. Key Technology Research about Omni-directional Mobile Platform Based on Mecanum Wheel. Dongfang Electric Review, 2013, 27(4): 7-11. (Chinese)

[3] HOU Bin, LI Ya-ming. The Mecanum Wheel Technology and its Application in Ship Wheel Device. Ship Engineering, 2011, 33(3): 84-87. (Chinese)

[4] LV Yong-jian, CONG Xin-yong, YANG Ming. Design of Mecanum Wheeled Omni-Directional Equipped Vehicle Driven by Stepper Motor. Small \& Special
Electrical Machines, 2013, 41(12): 14-16. (Chinese)

[5] Wang Yizhi. Research on Omni-directional Moving Technology for Building Environment, 2009. (Chinese)

[6] SHI Cai-hong, KANG Shao-hua, DUAN Xiu-bing. Development of Omni-directional Mobile System of Patient Transfer Robot. Automation and Instrumentation, 2009, 24(9): 5-8. (Chinese)

[7] Gong Shuang, Zhang Dongjun. Design and implementation of 2 DOF spherical inverted pendulum. Journal of Systems Engineering and Electronics, 2005, 16(1):123-127.

[8] Tlale N., de Villiers M. Kinematics and dynamics modelling of a Mecanum wheeled mobile platform. Mechatronics and Machine Vision in Practice, 2008. M2VIP 2008. 15th International Conference on. IEEE, 2008: 657-662.

[9] Zimmermann K., Zeidis I., Abdelrahman M. Dynamics of Mechanical Systems with Mecanum Wheels. Applied Non-Linear Dynamical Systems. Springer International Publishing, 2014: 269279.

[10] Tsai C. C., Wu H L. Nonsingular terminal sliding control using fuzzy wavelet networks for Mecanum wheeled Omni-directional vehicles. Fuzzy Systems (FUZZ), 2010 IEEE International Conference on. IEEE, 2010: 1-6.

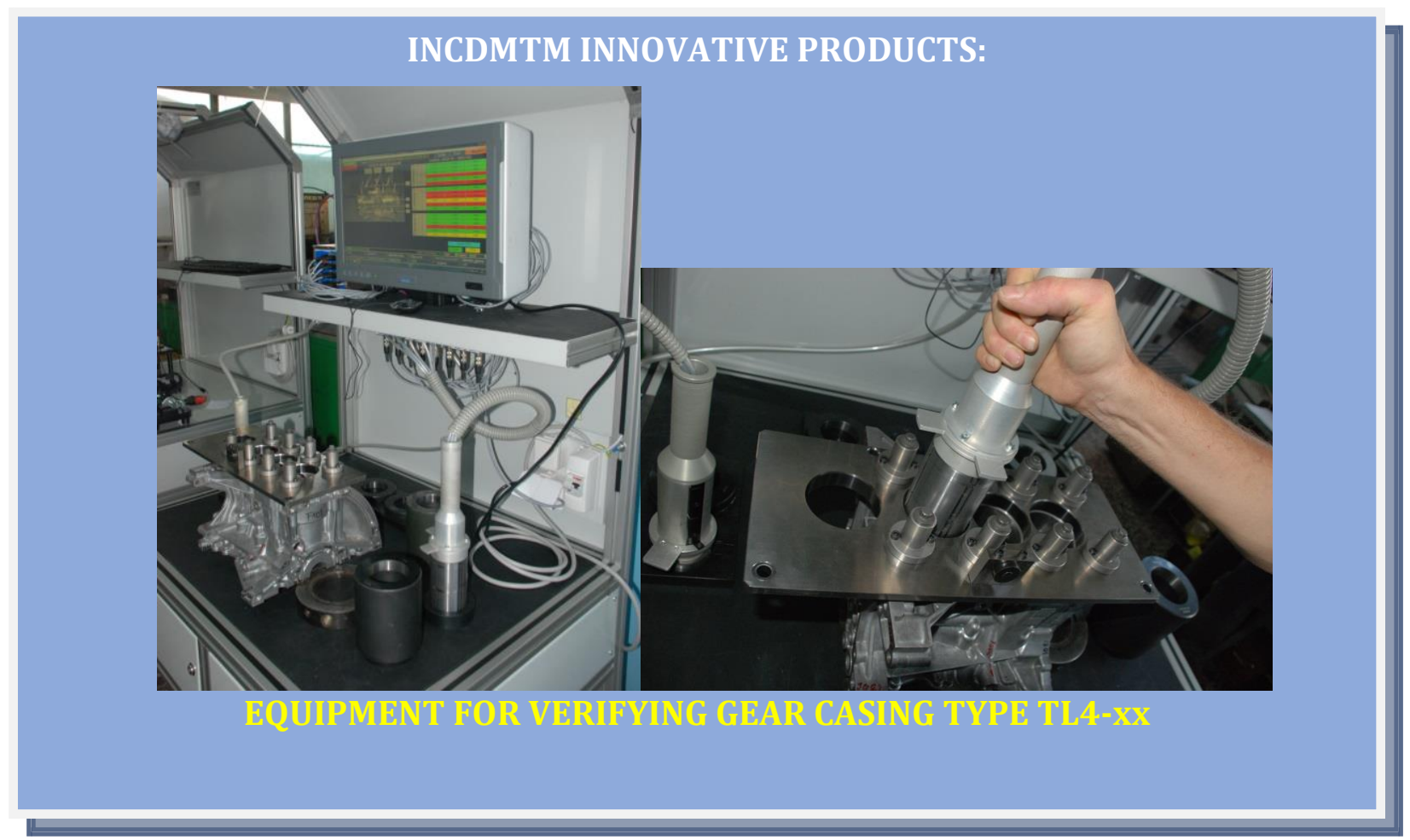

